\title{
On the generation of electric field and infrared radiation in aerosol clouds due to radon emanation in the atmosphere before earthquakes
}

\author{
V. A. Liperovsky ${ }^{1}$, C.-V. Meister ${ }^{2}$, E. V. Liperovskaya ${ }^{1}$, and V. V. Bogdanov $^{3}$ \\ ${ }^{1}$ Institute of Physics of the Earth of the Russian Academy of Sciences, Bolshaya Gruzinskaya 10, 123995 Moscow, Russia \\ ${ }^{2}$ Project Physics of Stellar and Planetary Atmospheres, An der Sternwarte 16, 14482 Potsdam, Germany \\ ${ }^{3}$ Institute of Cosmophysical Research and Radio Wave Propagation, Far Eastern Branch of the Russian Academy of Sciences, \\ 684034 Petropavlovsk-Kamchatsky, Russia
}

Received: 9 July 2008 - Revised: 18 August 2008 - Accepted: 25 August 2008 - Published: 28 October 2008

\begin{abstract}
The non-stationary Frenkel model of the generator of spikes of the local electric field with time scales of 1-100 min in the atmosphere near seismic fracture regions some days before strong earthqukes is analysed. The model suggests an aerosol cloud, an increased ionisation velocity (e.g. by radon emanation), and an upward flow of air. It was found that during times of earthquake activity (that means a few days before an earthquake), for realistic increases of the ionization intensity of the atmosphere, mosaic-likely occurring and disappearing pulses of local electric fields with intensities of the order of $10^{3}-3 \times 10^{3} \mathrm{Vm}^{-1}$ should be observable. These electric fields would also cause spikes of non-equilibrium IR emissions $(0.7-20 \mu \mathrm{m})$ and local spikes of the magnetic field. The authors propose to perform special complex Earth-based observations of the night-time emissions of the atmosphere in the IR region and of the magnetic as well as quasi-stationary electric fields at some points near to fracture regions in seismo-active belts.
\end{abstract}

\section{Introduction}

During the last decades, luminous phenomena and anomalous variations of the electric field in the near-Earth atmosphere before earthquakes were mentioned by various groups of scientists. But luminous phenomena before earthquakes occur rather seldom. According to the investigations of Papadopoulos (1999), taking into account also historical

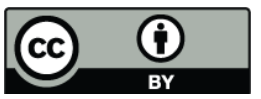

Correspondence to: C.-V. Meister (cvmeister@aip.de) sources of information since 2000 years, pulses of light - or so-called "fire" - was seen 5-6 days before earthquakes with magnitudes $M>6$ in one case out of 20 cases. Mostly the light lasted some seconds. If the electric fields of the period of earthquake activity are not strong enough to excite radiation in the visible region, it might happen that these fields are nevertheless strong enough to generate spikes of infrared (IR) emissions with wavelengths which are one-two orders larger. In such a case, the IR emissions may be considered as earthquake precursors.

The present work discusses a hypothesis on the local, nonstationary generation of an atmospheric electric field some days before earthquakes. There a strengthening of the ionization process in the near-Earth atmosphere by the occurrence of radon, and by an increase of the amount of aerosols and the local vertical air fluxes, is proposed.

\section{Local pulses of the atmospheric electric field - a pos- sible reason of an anomaly of the infrared emission}

Investigations of modifications of the quasi-constant electric field related to earthquakes have been already studied for some decades (Gokhberg et al., 1988; Morgunov, 1998; Vershinin et al., 1999; Pulinets et al., 2000; Mal'zev and Morgunov, 2005; Sorokin et al., 2006, 2007). In these works, anomalies of the temporal behaviour of the vertical component of the quasi-stationary electric field were analysed, which were connected with earthquakes for which already known meteorological phenomena were not obtained. It was found that rather long-lasting anomalies of the electric field and vertical currents appear a few hours, or only tens of minutes, before earthquakes at distances from the epicentres not

Published by Copernicus Publications on behalf of the European Geosciences Union. 
larger than $200 \mathrm{~km}$. The long-lasting anomalies were not connected with visible atmospheric sources, and the intensity of the anomalous variations did not directly depend on the parameters of the earthquake (Rulenko, 2000). The authors of the present work think that, in this connection, anomalies of the electric field and vertical currents some days before earthquakes were not yet analyzed sufficiently. There has yet to be performed a statistical analysis taking into account a larger number of earthquakes.

Recent measurements and analyses of variations of the vertical electric field connected to earthquakes (and also simultaneously of other parameters characterizing the electrical activity in the point of observation) are described in (Mikhailov et al., 2004; Smirnov, 2005; Mikhailov et al., 2005). The observations showed that the anomalies of the daily changes of the quasi-stationary vertical electric field in the atmosphere near the Earth's surface before earthquakes have the form of spikes. Most often the electric field decreases by different values and during different time intervals. The anomalies of the electric field before and directly after the earthquakes are often one-two orders of magnitude larger than the background values. The most probable value of the amplitude of the spikes is $100-300 \mathrm{Vm}^{-1}$, and the most probable duration time of the anomaly amounts to 30 100 min.

Considering atmospheric electric phenomena before earthquakes, aerosols play an important role. The electrical conductivity and the electric field of the atmosphere change during variations of the density and the kinds of aerosols. Because of the breaking of dust particles, the friction between them, and the attraction of small ions to the dust, aerosol particles become charged. Such modifications of aerosols in the near-earth atmosphere a few hours before earthquakes are considered in (Alekseev and Aksenov, 2003; Grishin et al., 2003). At night, the condensation of water vapour on charged aerosol particles is very probable. Thereat, large aerosol particles are more often negatively charged, and smaller particles are above all positively charged. The occurrence of large negative charges at altitudes above the surface larger than some tenths of meters, causing an electric field of opposite polarity, may be interpreted by the mechanism formerly used to explain the formation of the Frenkel generator, which results into local currents and IR emissions (Liperovsky et al., 2005).

If in the atmosphere radioactive material occurs, then the densities of positively and negatively charged particles grows. Thus the influence of aerosol particles on the atmospheric electric field increases correspondingly. Anomalies of the electric field before earthquakes were related by several authors to the increase of the electrical conductivity of the air by radon emanation. One model explaining the generation of the anomalous electric field before earthquakes was proposed by Pulinets et al. (2002) and developed in (Pulinets and Boyarchuk, 2004). This model takes also the strengthening of the ionization during the outflow of radon from the Earth's core and by the hydrotation process into account. Hydrotation - that is the attraction of water molecules to the newly formed ions and ion clusters, which protect the charged particles from recombination and make them quasi-stable. Another model, which takes the increase of the number of aerosols and the strengthening of ionization processes into account too, is presented in the series of works (Heinke et al., 1995; Sorokin and Tshmyrev, 2002). Omori et al. (2006) considered the effects of radon on the preseismic electromagnetic phenomena by use of the data on the anomalous concentration of atmospheric radon measured at the time of the Kobe earthquake.

Thus, the occurrence of non-stationary aerosol clouds and radioactive material results into non-stationary pulses of electrical conductivity and electric field.

\section{Models of possible structures of charged aerosols and some estimations}

Let us consider two simple models of the generation of electric fields. Let us suggest that an aerosol cloud is injected into the near-Earth, locally heated atmosphere above a fracture region and that it gets into an upward directed flux of air. Thereat, the characteristic time scale of the duration of the outflow and upward propagation of the cloud may be of the order of tens of seconds. This characteristic time scale is much smaller than the evolution time of the cloud, which is larger than some tenths of minutes - or even larger than one hour.

Within the frame of the first model, at the initial moment of time, an aerosol cloud of the form of a sphere with radius $a_{o}$ is situated in the atmosphere. It is assumed, that two types of aerosols exist - large ones with a radius $R=10 \mu \mathrm{m}$ and a density $N_{R}$, and small ones with radius $r=1 \mu \mathrm{m}$ and density $N_{r}$. Further, it is suggested, that, in the initial moment of time, radon is put into the aerosol cloud, and a homogeneous ionisation occures. Large aerosol particles are negatively charged (Smirnov, 1992), but small aerosol particles are positively charged. The vertical velocity of the small particles is much smaller than that of the large ones, which equals a few $\mathrm{cm} \mathrm{s}^{-1}$. Within the present simple model, it is suggested that ionization and additional charging of the aerosols end quickly, and the large aerosol particles, charged negatively, fall quickly down and have a relative velocity $U_{-}$ directed downwards, while the small particles are practically at rest.

The above described system is a "Frenkel generator" of a local electric field (Frenkel, 1949; Shishkin, 1954). The intensity of the electric field reaches its maximum in the middle of the line linking the centres of the differently charged aerosols. It linearly increases up to the moment of time $t^{*}$, at which the negatively charged sphere has fallen down so much, that the differently charged spheres are separated. As result, at the moment $t^{*}=2 a_{o} / U_{-}$, two differently charged 
aerosol spheres exist, and the maximum electric field occurs in the point of contact of the both spheres.

Further, when the spheres depart from one another, the intensity of the electric field decreases again. The characteristic time scale of the existence of the "Frenkel generator" and the corresponding local current system equals $\Delta t=2 a_{o} / U_{-}$. The maximum value of the electric field as function of the density of the positively charged particles $\rho_{+}$and the characteristic dimensions of the aerosol cloud may be presented by

$E_{\max }=2 E_{\mathrm{ball}}=\frac{V \rho_{+}}{2 \pi \varepsilon_{o} a_{o}^{2}}=\frac{V \rho_{+}}{2 \varepsilon_{o} S}$

in the point of contact ( $S$ describes the area of the projection of the sphere on the horizontal plane),

The second useful model of the generation of the electric field is the model of the "plane capacitor" (see e.g. Liperovsky et al., 2005) In this case the maximum electric field equals

$E_{\mathrm{cond}}=\frac{z_{o} \rho_{+}}{\varepsilon_{o}}=\frac{x_{o} y_{o} z_{o} \rho_{+}}{x_{o} y_{o} \varepsilon_{o}}=\frac{V \rho_{+}}{S \varepsilon_{o}}$,

here $z_{o}$ is the maximum dimension of the vertical shift, or "thickness of the capacitor plate", $x_{o}$ and $y_{o}$ - the characteristic horizontal dimensions of the cloud, and $\rho_{+}-$the density of the charged particles. The characteristic time scale of the existence of the maximum field is determined by the minimum horizontal dimensions of the cloud

$\Delta \tau \sim \frac{\sqrt{\min \left(x_{o}^{2}, y_{o}^{2}\right)}}{U_{-}}$.

In all cases, the amplitude of the spike of the vertical local electric field depends on the mean density of the positive charges in the projection area $V \rho_{+} / S$. Thereat, the charges are positive in the upper part of the cloud and negative in the lower part. One of the possible real situations is shown in Fig. 1.

The maximum electric fields obtained within the two models differ one from another only by a factor 2 . This justifies the estimation of the electric field without consideration of the geometry of the distribution of the aerosols and the geometry of their ionisation. The "existence time" of the maximum electric field $\tau_{o}$ may be found assuming that the maximum dimension of the vertical shift $z_{o}$ almost equals the horizontal dimension of the system, $\tau_{o} \sim R_{\text {horiz }} / U_{-}$. For estimates of the maximum electric field in the "Frenkel generator" one may use $E=V \rho_{+} / 2 S \varepsilon_{o} . S$ is the projection area of the generator on the Earth's surface.

In cases of sufficiently large electric field generation areas near the points of measurements, the observed anomalies of the electric field may have values much larger than the mean values, and they may occur in bay-form with a duration of 30-100 min. In more realistic models, the further continuation of the ionisation process has also to be taken into accout, and one finds a somewhat longer duration time. If the

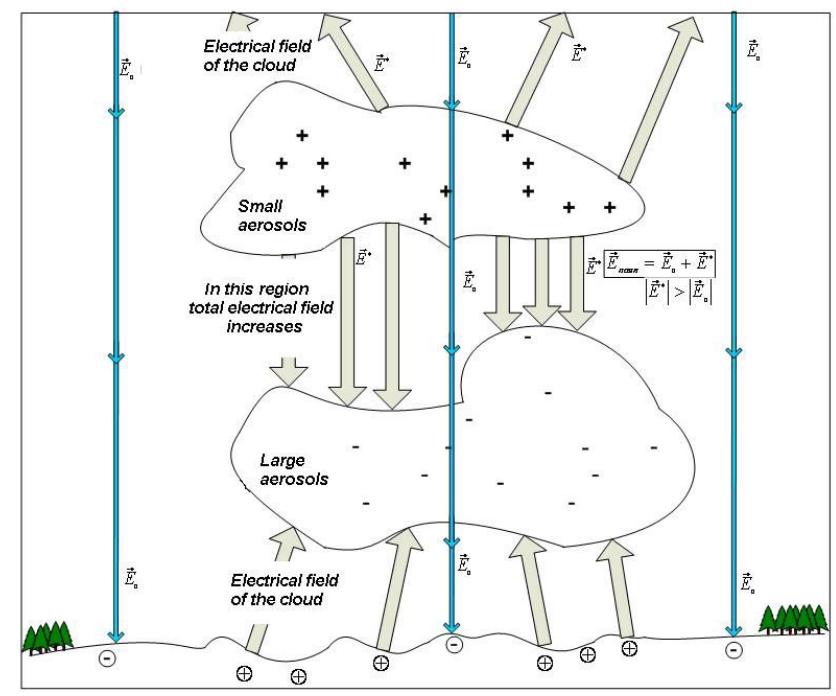

Fig. 1. One of the possible real situations of the charge separation in aerosol clouds. The initial main electric field $\boldsymbol{E}_{O}=130 \mathrm{~V} / \mathrm{m}$ is directed towards the Earth's surface. The electric field disturbance $\boldsymbol{E}^{*}$ occurs because of the charge separation. The resulting electric field $\boldsymbol{E}=\boldsymbol{E}_{o}+\boldsymbol{E}^{*}$ above and below the cloud may be smaller than $\boldsymbol{E}_{o}$, it may be even directed oppositely to $\boldsymbol{E}_{o}$.

distance between the electric field generation region and the observation point is larger, one finds electric fields larger than the background fields only during shorter time intervals.

Let us consider possible non-stationary processes taking the atmospheric electrical conductivity into account. It is assumed that in the initial moment of time $t=0$, in the atmosphere exists already a cloud of aerosols, and the ionization intensity $q_{i}$ is much increased by the growth of radon concentration. It is taken $q_{i} \approx 10^{2}-10^{4} \mathrm{~cm}^{-3} \mathrm{~s}^{-1}$ (Wick and Singh, 1994; Heinke et al., 1995; Pulinets and Boyarchuk, 2004), which is much larger than the average background value $q_{i}^{o} \approx 10 \mathrm{~cm}^{-3} \mathrm{~s}^{-1}$.

Let us consider the system of equations describing the ionisation and recombination of the ions $n^{+}$and $n^{-}$, and the collisions of the ions with the aerosols causing the modification of the charge of both the small aerosols with $N_{a}^{+}$and the large aerosols with $N_{a}^{-}$(Smirnov, 1992; Pulinets et al., 2000). If the concentration of aerosols is sufficiently large and the process limiting the increase of the ion concentration is the collisional charge transfer from the ions to the aerosols, one has

$$
\frac{\partial n_{i}^{ \pm}}{\partial t}=q_{i}-n_{i}^{ \pm} \beta\left(N_{a}^{+}+N_{a}^{-}\right),
$$

where $\beta$ is the recombination coefficient for the charge transfer from ions to aerosols. For estimations, one may use $\beta=2 \times 10^{6} \mathrm{~cm}^{3} \mathrm{~s}^{-1}$. Further, it is assumed $Q^{+} N_{a}^{+}=Q^{-} N_{a}^{-}$. Then, for sufficiently large aerosol concentrations with $\alpha\left(n_{i}^{ \pm}\right)^{2} \ll n_{i}^{ \pm} \beta N_{a}^{+}\left(\alpha \approx 10^{-7} \mathrm{~cm}^{3} \mathrm{~s}^{-1}\right.$ is the recombination 
Table 1. Near-IR spectral lines which should be of importance for earthquake prediction.

\begin{tabular}{llllll}
\hline ozon $\mathrm{O}_{3}$ & 4.75 & 55.75 & 9.1 & 9.6 & 10.5 \\
& 14.1 & & & & \\
carbon dioxide $\mathrm{CO}_{2}$ & 2.7 & $\mathbf{4 . 2 - 4 . 4}$ & 4.8 & 5.2 & 9.4 \\
& $\mathbf{1 0 . 5}$ & $\mathbf{1 3 . 5}-\mathbf{- 1 8 . 4}$ & & & \\
nitrogen dioxide $\mathrm{NO}_{2}$ & 9.6 & 17.0 & & & \\
methan $\mathrm{CH}_{4}$ & 3.3 & $\mathbf{7 . 7}$ & & & \\
\hline
\end{tabular}

coefficient for the collisional recombination of positive and negative ions), one finds $n_{i}^{ \pm}=q_{i} /\left(\beta N_{a}^{+}\right)$.

For densities of positively charged aerosols $N_{a}^{+}=10^{4} \mathrm{~cm}^{-3}$, one finds $n_{i}^{ \pm}=0.5 \times 10^{4} \mathrm{~cm}^{-3}$, that means the ion concentration is smaller than at times without aerosols and increased ionization. Thus, for one and the same velocity of ion formation, one finds lower ion concentrations if there exist aerosols additionally. Consequently, the electrical conductivity decreases and the quasi-constant electric field grows. Let us consider the case of increased ion formation at simultaneous growth of the aerosol concentration in more detail within the frame of the model of the plane capacitor. On the one side, the electric field increases. because of the upward motion of the negatively charged aerosols, but, on the other side, it decreases by the compensating electrical current between the clouds,

$\varepsilon_{o} \frac{\partial E_{l}(t)}{\partial t}=\left(U_{-} Q^{-} N_{a}^{-}-\lambda_{o} \frac{n}{n_{o}} E_{l}(t)\right)-\left(\frac{\partial E}{\partial t}\right)_{\text {horiz }}$.

$\lambda_{o}$ is the electrical conductivity of the near-surface atmospheric air layer, $Q^{-}-$the value of the charges of the negatively charged aerosols. The last term in the formula describes the change of the electric field by horizontal electric currents connected with the divergency of the wind velocity. Taking also the force balance

$\frac{4}{3} \pi R^{3} \rho_{a} g-6 \pi R \eta U_{-}-Q^{-} E_{l}=0$

into account ( $\rho_{a}$ - aerosol density, $\eta$ - viscosity of air, $g-$ acceleration due to gravity), one finds (neglecting horizontal electric currents) for the maximum electric field

$E_{l M}=\frac{2 R^{2} \rho_{a} N_{a}^{-} Q^{-} g n_{o}}{9 \eta \lambda_{o} n}$.

The time of the field increase $\tau_{1}$ may be estimated by the characteristic maxwellian relaxation time

$\tau_{1} \approx \frac{\varepsilon_{o} n_{o}}{\lambda_{o} n}=\frac{\varepsilon_{o} n_{o} \beta N_{a}^{+}}{\lambda_{o} q_{i}}$.
Performing further estimations, here the values for an undisturbed electrical conductivity of the air at the earth's surface of $\lambda_{o}=10^{-14} \mathrm{Sm} \mathrm{m}^{-1}$, an air viscosity $\eta=1.8 \cdot 10^{-5} \mathrm{~kg} \mathrm{~m}^{-1} \mathrm{~s}^{-1}$, and an aerosol mass density $\rho_{a}=2.5 \cdot 10^{3} \mathrm{~kg} \mathrm{~m}^{-3}$ given by (Kondratjev and Poznjakov 1981) are used. Chosing for the concentration of the large aerosols $N_{a}^{-}=10^{9} \mathrm{~m}^{-3}$, the charge of the large aerosols $Q^{-}=2000 e$ ( $e$ - electron charge), $g=10 \mathrm{~ms}^{-2}$, $n_{o}=\sqrt{q_{i}^{o} / \alpha}=10^{4} \mathrm{~cm}^{-3}, n=0.5 \cdot 10^{4} \mathrm{~cm}^{-3}, n / n_{o}=0.5$, one obtaines for the maximum electric field $E_{l M}=2 \cdot 10^{6} \mathrm{~V} \mathrm{~m}^{-1}$ (breakdown at $3 \cdot 10^{6} \mathrm{~V} \mathrm{~m}^{-1}$ ).

The total time of the occurrence of the spike of the electrical field is the sum of the time of increase of the electric field $\tau_{1}$, the time $\tau_{2}$ during which the positively and negatively charged aerosol clouds cover one another ("plateau time"), and the relaxation time after the end of the separation of differently charged aerosols $\tau_{3} \approx \tau_{1}$. The local relaxation time $\tau_{1}$ decreases if the ionization velocity $q_{i}$ increases, but it increases (and the ion concentration $n$ decreases) if the aerosol concentration $N_{a}^{+}$grows. If the main process is the increase of the ionization velocity $q_{i}$, then one may find decreased values of the relaxation time of the order of 1-10 min.

Under conditions with a high concentration of noncharged aerosols, the usual value of the electrical conductivity may decrease, for instance, by one order. Then the electric field in the system increases, but not so strongly because of the finite horizontal dimensions of the "capacitor" and the influence of the environment of the aerosol cloud.

The analysis of realistic parameters showed, that, for the problem of earthquake prediction, cases with an ionization intensity of $q_{i} \sim\left(3 \times 10^{2}-3 \times 10^{4}\right) \mathrm{cm}^{-3} \mathrm{~s}^{-1}$ are the most interesting ones. One would observe amplitudes of the electric field disturbances of $0.3 \times 10^{5}-6 \times 10^{5} \mathrm{~V} \mathrm{~m}^{-1}$. The non-stationary change of the electric field causes a spike of the non-equilibrium IR emission, which might last 3060-100 min, if the vertical thickness of the aerosol cloud amounts to some $100 \mathrm{~m}$ and the dimension of the aerosols equals a few tenths of micrometers.

Of special importance are the non-equilibrium IR emissions caused by the electron or ion acceleration within the free path of the particle in the electric field, and the excitation of spectral lines of the main atmospheric components by the accelerated particles. The heating of the atmosphere connected with such phenomena is rather weak, but it influences the equilibrium radiation which is usually registered by satellites (Zuev, 1980).

As for the earthquake predictions IR emissions with wavelengths of $0.7-20 \mu \mathrm{m}$ seem to be of importance, one may conclude that the electric field spikes should have amplitudes of $10^{3}-10^{4} \mathrm{~V} \mathrm{~m}^{-1}$. In the near-surface layer of the Earth, there should occur some smaller electric fields of 200 $500 \mathrm{~V} \mathrm{~m}^{-1}$. 
Table 2. Estimates of local vertical electric field changes $\Delta E$.

\begin{tabular}{lll}
$\lambda$ wavelength of IR emission $[\mu \mathrm{m}]$ & $W=h c / \lambda$ & $\Delta E$ \\
& $\mathrm{eV}$ & $\mathrm{V} / \mathrm{m}$ \\
0.7 (red border line of visible spectrum) & 1.8 & $2.5 \times 10^{5}$ \\
$7.7\left(\mathrm{CH}_{4}\right.$-increase a few days before the earthquake) & 0.17 & $2.3 \times 10^{4}$ \\
$10.5\left(\mathrm{CO}_{2}\right.$, line used by $\mathrm{CO}_{2}$-laser) & 0.13 & $1.7 \times 10^{4}$ \\
15.0 (absorption and expected emission interval of $\left.\mathrm{CO}_{2} 13.5-18.4\right)$ & 0.08 & $1.2 \times 10^{4}$ \\
\hline
\end{tabular}

The ionization energy in the atmosphere $W_{o} \approx q E_{o} L_{f}$ is of the order of $15 \mathrm{eV}$, the breakdown electric field $E_{o}$ amounts to $3 \times 10^{6} \mathrm{~V} \mathrm{~m}^{-1}$, and the mean free path of the particles is $L_{f} \approx 7 \mu \mathrm{m}$. Under the condition that the electric field is smaller than the breakdown one, the energy transferred to a particle in the constant electric field is of the order of the quantum of the IR emission energy $q E L_{f} \approx W \approx h c / \lambda$, where $\lambda$ designates the wavelength of the photon in the IR part of the spectrum.

During times of earthquake activity in seismo-active regions, there occur chemical and carbon-isotop instabilities of the currents of natural gases (Voitov and Dobrovolskii, 1994). In comparison with non-seismo-active regions, the density of methan $\mathrm{CH}_{4}$ in the near-earth atmosphere above tectonically destructed structures increases, on the average, 1.5-2 times. An analogous growth was found for carbon dioxide $\mathrm{CO}_{2}$. Thus, as a consequence of the outflow of gases out of the Earth's surface, above all of $\mathrm{CO}_{2}$ and $\mathrm{CH}_{4}$, the emission spectrum of the air is slightly modified. Emissions in the interval of $2-15 \mu \mathrm{m}$ are intensified, especially the spectrum at $4.3 \mu \mathrm{m}, 7.7 \mu \mathrm{m}$, and $15 \mu \mathrm{m}$.

Consequently, observing IR emissions in nights without thunderstorms, one may obtain mosaic-like structures of electric fields generated by a Frenkel-typ mechanism. The occurrence of these structures, and also the strengthening of some spectral lines caused by the additional outflow of $\mathrm{CH}_{4}$ and $\mathrm{CO}_{2}$, allow to make conclusions about a growing probability of an earthquake in the next days. The corresponding observations could be performed with a spectrometer with circular registration, which should be situated on the top of a mountain.

In Table 1, near-IR absorption and expected emission intervals of lines and spectral lines are shown which correspond to wavelengths which are about $0.5-1.5$ orders of magnitude larger than the red border line, $\lambda \approx(2.2-22) \mu \mathrm{m}$. The boldface numbers in Table 1 designate the spectral intervals and spectral lines which are most interesting for earthquake prediction. Analysing this IR emissions, one should obtain information about spikes of the atmospheric electric field connected to earthquakes. It is assumed that the accelerated by the electric field spike electrons and ions collide with molecules, excite them, and the molecules emit IR radiation within special intervals of the wavelength. In Table 2, estimates of the local vertical electric field changes $\Delta E$ are presented, which should cause emissions in the considered spectral intervals or at the considered lines. Further the energies of the photons emitted at the mean wavelength of the intervals are given.

In the cases of $\mathrm{CO}_{2}$ and $\mathrm{CH}_{4}$, the mass density of the gases is almost constant in the earth's atmosphere within $30 \mathrm{~km}$ of altitude above the surface. The density of $\mathrm{CO}_{2}$ amounts to about $3.3 \times 10^{-2} \%$, and $\mathrm{CH}_{4}$ contributes to $1.6 \times 10^{-4} \%$ to the air. It is known that the differences between maximum and minimum concentrations of these gases may be some times larger than their mean values. Presenting in Table 2 estimates of the electric field spikes it has to be underlined that the mean free path of the molecules and electrons may be described by a distribution function of the type $f(\lambda) \sim \exp \left(-\lambda / \lambda_{o}\right)\left(\lambda_{o}\right.$ is the mean wavelength). Thus, the electric field may be about half an orders of magnitude larger or smaller than the field given in Table 2. Thus, here one suggests to register electric fields of the order of $3 \times 10^{3} \mathrm{~V} \mathrm{~m}^{-1}$. If the electric fields are generated at larger altitudes of 5$10 \mathrm{~km}$, and the free paths of the particles in the electric field are accordingly larger, than the IR emission may occur within weaker electric fields of the order or smaller than $10^{3} \mathrm{~V} \mathrm{~m}^{-1}$.

\section{Conclusions}

The non-stationary Frenkel model of the generator of spikes of the local electric field with time scales of 1-100 min in the atmosphere near seismic fracture regions some days before strong earthqukes is analysed. The model suggests an aerosol cloud, an increased ionisation velocity (e.g. by radon emanation), and an upward flow of air.

Indeed, this mechanism may work in Japan and on Kamchatka. The authors assume that it is also possible in Tashkent. They underline that in these regions, there exist ionospheric effects which seem to be caused by nonstationary electric fields in the atmosphere which are the consequence of radon emanation before earthquakes. One example of such an effect is the decrease of the critical foF2 frequency.

At large altitudes above the surface of the oceans and in regions with weak industrial activity one finds only a small amount of aerosols of technical origin. At low humidity, water aerosols also do not form. Such conditions occur, for 
instance, in the region around Alma-Aty. Thus, the described mechanism of the generation of electric fields seems not to work there. It shoud be mentioned that, according to the data of the vertical ionospheric sounding station Alma-Aty, a decrease of foF 2 was indeed also not obtained there before earthquakes.

Mosaic-likely occurring and disappearing pulses of local electric fields should cause corresponding spikes of nonequilibrium IR emissions $(0.7-20 \mu \mathrm{m})$ and local spikes of the magnetic field. Although anomalies of the equilibrium IR emissions have been observed several times by satellites above fracture regions at night before earthquakes, shorttime local spikes of the magnetic field and non-stationary spikes of the non-equilibrium night-time IR emissions with specific specta seem not to have been observed up to now.

It has to be underlined that there work different mechanisms of the generation of electric field in the near-Earth atmosphere, atmospheric and lithospheric (basing on the formation of fractions) ones. And all these mechanisms should cause spikes of the non-equilibrium IR emission. The investigation of the complex of such emissions should help to improve the earthquake prediction. But practically, one has yet to improve the registration of IR emissions - there are necessary continuous measurements of IR spectra.

Helpful will also be special complex Earth-based observations of the night-time emissions of the atmosphere in the IR region and of the magnetic and quasi-stationary electric fields at some points near to fracture regions in seismo-active belts. To understand the physical phenomena, at the same time also standard vertical sounding programmes have to be performed.

Edited by: P. F. Biagi

Reviewed by: G. Martinelli and another anonymous referee

\section{References}

Alekseev, A. S. and Aksenov, V. V.: On the elctromagnetic field in the hearth zone of earthquakes, Doklady Akademii Nauk, 392(1), 101-110, 2003.

Frenkel, Y. I.: Theory of atmospheric electricity phenomena, GITTL, Moscow-Leningrad, 1949.

Gokhberg, M. B., Morgunov, V. A., and Pokhotelov, O. A.: Seismoelectromagnetic phenomena, Nauka, 1988.

Grishin, A. N., Matvienko, G. G., Alekseev, V. A., Alekseeva, N. G., Dontshenko, V. A., and Vodnev, S. A.: Aerosol and electric characteristics of the atmosphere in seismo-dangerous regions, Tomsk Institute of Atmospheric Optics SO RAN, preprint No. 6, 2003.

Heinke, J., Koch, H., and Martinelli, G.: $\mathrm{CO}_{2}$ and radon measurements in the Vostland (Germany) - a contribution to earthquake prediction research, Geophys. Res. Lett., 22, 749-774, 1995.

Kondrat'jev, K. J. and Poznjakov, D. V.: Aerosol models of the atmosphere, Nauka, Moscow, 1981.

Liperovsky, V. A., Meister, C.-V., Liperovskaya, E. V., Davidov, V. F., and Bogdanov, V. V.: On the possible influence of radon and aerosol injection into the atmosphere and ionosphere before earthquakes, Nat. Hazards Earth Syst. Sci., 5, 783-789, 2005, http://www.nat-hazards-earth-syst-sci.net/5/783/2005/.

Mal'zev, S. A. and Morgunov, V. A.: On the physical model of disturbances of the electric field of lithospheric nature in the atmosphere and EMI, Fizika Zemli, 9, 65-73, 2005.

Mikhailov, Y. M., Mikhailova, G. A., Kapustina, O. V., Buzevich, A. V., and Smirnov, S. E.: Power spectrum features of near-Earth atmospheric electric field in Kamchatka, Ann. Geophys., 47(1), 237-245, 2004.

Mikhailov, Y. M., Mikhailova, G. A., Kapustina, O. V., Buzevich, A. V., and Smirnov, S. E.: Peculiarities of atmospheric noise occurring during variations of the quasi-static field in the near-Earth atmosphere of Kamchatka, Geomagnetizm i Aeronomiya, 45(5), 690-705, 2005.

Morgunov, V. A.: Electrical phenomena before the Shikotan earthquake and its aftershocks, Dokl. RAN, 3359(1), 102-105, 1998.

Omori, Y., Yasuoka, Y., Nagahama, H., Kawada, Y., Ishikawa, T., Tokonami, S., and Shinogi, M.: Anomalous radon emanation linked to preseismic electromagnetic phenomena, Nat. Hazards Earth Syst. Sci., 7, 629-635, 2007, http://www.nat-hazards-earth-syst-sci.net/7/629/2007/.

Papadopoulos, G. A.: Luminous and fiery phenomena associateed with earthquakes in the East Mediterranean, Atmospheric and ionospheric electromagnetic phenomena associated with earthquakes, edited by: Hayakawa, M., Tokyo, TERRAPUB, 559575, 1999.

Pulinets, S. A., Boyarchuk, K. A., Hegai, V. V., and Karelin, A. V.: Conception and model of seismo-ionosphere-magnetosphere coupling, Seismo-Electromagnetics: Lithosphere-AtmosphereIonosphere Coupling, edited by: Hayakawa, M., Tokyo, TERRAPUB, 353-361, 2002.

Pulinets, S. A., Boyarchuk, K. A., Hegai, V. V., Kim, V. P., and Lomonosov, A. M.: Quasielectrostatical model of atmospherethermosphere-ionosphere coupling, Adv. Space Res., 26(8), 1209-1218, 2000.

Pulinets, S. A. and Boyarchuk, K. A.: Ionospheric precursors of earthquakes, Springer, Berlin, Heidelberg, New York, 2004.

Rulenko, O. P.: Operative precursors of earthquakes in the electricity of the near-Earth atmosphere, Vulcanology and Seismology, 4, 57-68, 2000.

Shishkin, N. S.: Cloud, precipitation and thunderstorm electricity, GITTL, Moscow, 1954.

Smirnov, V. V.: Ionisation in the troposphere, Gidrometeoizdat, St. Petersburg, 1992.

Smirnov, S. E.: Peculiarities of negative anomalies of the quasistatic electric field in the near-Earth atmosphere on Kamchatka, Geomagnetizm i Aeronomiya, 45(2), 282-287, 2005.

Sorokin, V. M. and Chmyrev, V. M.: Electrodynamic model of ionospheric precursors of earthquakes and of some other types of catastrophies, Geomagnetizm i Aeronomiya, 42(6), 820-830, 2002.

Sorokin, V. M., Yaschenko, A. K., Chmyrev, V. M., and Hayakawa, M.: DC electric field amplification in the mid-latitude ionosphere over seismically active faults, Physics and Chemistry of the Earth, 31, 447-453, 2006.

Sorokin, V. M., Yaschenko, A. K., and Hayakawa, M.: A perturbation of DC electric field caused by light ion adhesion to aerosols during the growth in seismic-related atmospheric radioactivity, Nat. Hazards Earth Syst. Sci., 7, 155-163, 2007, 
http://www.nat-hazards-earth-syst-sci.net/7/155/2007/.

Vershinin, E. F., Buzevitch, A. V., Yumoto, K., Saito, K., and Tanaka, Y.: Correlation of seismic activity with electromagnetic emissions and variations in Kamchatka region, Atmospheric and Ionospheric Phenomena Associated with Earthquakes, edited by: Hayakawa, M., TERRAPUB, Tokyo, 513-517, 1999.
Vojtov, G. I. and Dobrovol'skii, I. P.: Chemical and carbon-isotop instabilities of flows of natural gases in seismo-active regions, Fizika Zemli, 3, 20-31, 1994.

Wick, H. S. and Singh, B.: Radon recording of Uttarkashi earthquake, Geophys. Res. Lett., 21, 737-740, 1994.

Zujev, V. E.: Propagation of visible and infrared waves in the atmosphere, Soviet Radio, Moscow, 1980. 